\title{
Surgical site surveillance: Quality improvement or waste of time?
}

$\mathrm{S}$ URGICAL SITE INFECTION (SSI) IS A RELATIVELY INFREQUENT complication of surgery, yet it is a major medical problem because thousands of operations are done weekly across Canada. ssi has several possible outcomes, including the morbidity related to the infection per se, secondary complications such as ventral hernia and necrotizing soft tissue infection and, rarely, death. The prevention of ssi requires good judgement in patient selection and preparation, meticulous surgical technique and judicious use of prophylactic antimicrobial agents. For clean operations, infection rates as low as $1 \%$ have been reported (1).

In the early 19th century infection at the operative site was exceedingly common, and the results were usually disastrous; for example, limb amputation carried a mortality rate of 30 to $50 \%$. Pasteur's work on the microbial cause of infection and the studies of Semmelweis and Lister led to the use of antisepsis, then asepsis, in the operating theatre. After the discovery of safe general anesthesia in the 19th century, surgery performed at frantic speed to reduce the duration of pain gave way to the precepts of Halsted and other modern pioneers, who demonstrated that meticulous surgical technique improved the chance of a successful outcome. Clean surgical procedures had become safe. In the $1960 \mathrm{~s}$ a similar but less dramatic decrease in infection rates was seen in clean contaminated operations as prophylactic antimicrobial agents were successfully used.

It is evident that past reductions in SSI rates have occurred not by gradual decrements but by relatively precipitous drops in response to important discoveries. Current ssi rates seem too low to be reduced drastically, but it is the aim of surgeons and infection control teams to continue to decrease their incidence. Can 'zero infection' rates be achieved or has an irreducible minimum been reached? Most practitioners interested in infection control believe that efforts at quality improvement will continue to reduce SsI rates by the same mechanism as quality improvement programs used in industry: determination of incidences of failures followed by attempts to "do better next time". Indeed, growing numbers of reported studies have shown that calculation of SSI rates and confidential reporting of those rates to individual surgeons lead to reductions in subsequent rates of SSI $(1,2)$. Such studies have become show- pieces in the quality improvement movement and have led to sSI surveillance being mandated by accreditation bodies.

The study by Taylor et al (pages 263-267) in this issue of the Journal is another attempt to demonstrate that SSI surveillance reduces infection rates following operations. The authors point out that, despite the demonstrated success of ssi surveillance, a minority of Canadian hospitals has continuous surveillance programs. They found that, with the exceptions of neurosurgery and orthopedics, wound and other operative site infections diminished between 1987 and 1993 at the University of Alberta Hospitals. As the study progressed, the authors improved their program by reporting risk-adjusted wound infection rates compared with National Nosocomial Infection Study rates. Although not stated by the authors, implicit in ssi surveillance programs is the competitive desire of surgeons to improve their recorded statistics. The authors conclude that their study supports the value of ssi surveillance.

Although I participate in a similar ssi surveillance program at The Wellesley Hospital in Toronto, Ontario and believe that my competitive edge is indeed sharpened by reports of infections I have caused, I can find little objective evidence to support the use of ssi surveillance. The study of Taylor et al has flaws that typify, on a large scale, our inability to prove that ssi surveillance does what it is supposed to do. Although it appears that SSI rates have diminished at the University of Alberta Hospitals, they did not do so on all surgical services; no reason could be found for the sSI rate increase on orthopedics. One would like to believe that the ssi rate reductions on the other services occurred because of feedback to surgeons, but the 'improvements' might have been temporary downward trends in periodically fluctuating SSI rates. Positive results get published and negative results stay home. For the five or 10 published studies that have shown ssi rate reductions with surveillance, perhaps an equal number were never published because of failures to achieve ssi rate reductions because surveillance was conducted during periodic upturns or stable periods of ssi rates. One would hope that SSI rate reductions in Dr Taylor's study resulted from better surgical care, yet they may simply have been the product of less rigorous surveillance, ie, decreased availability of the infection control practitioner 
from 1992 onwards and reduced lengths of hospital stay after operation, leading to fewer in-hospital diagnoses of Ssi. Others have reported that half of all SSI now occur following discharge from hospital $(3,4)$, and these were not captured by the authors. The apparent cost saving of surveillance can be challenged when the SSI rate reductions may be more apparent than real and the cost of an infection control practitioner has not been included. These pitfalls are not unique to Dr Taylor's study. They are characteristic of 'inclusive' SSI surveillance programs, which calculate a 'numerator' of infection cases divided by a much larger 'denominator' consisting of the total number of operative procedures in a given category.

Inclusive ssi surveillance has been challenged by Dr James Lee, a surgeon at the University of Minnesota and a member of the Surgical Wound Infection Task Force convened by the Society of Hospital Epidemiology of America (2). Dr Lee believes that we waste resources in calculating the denominator, which consists almost completely of cases in which infection does not occur. Rather, he advocates we concentrate on the numerator - review and analyze cases in which infection did occur. and ask ourselves what went wrong (5, personal communication). At The Wellesley Hospital, we have done this by analyzing one variable that is easy to assess: the timing of administration of prophylactic antibiotics. We found that in about a quarter of cases of ssi, a prophylactic antimicrobial agent was given too soon before operation to have any use; similar findings have been reported in the literature (6). Cases of SSI should be discussed at regular morbidity rounds in every surgical division. This is not done universally in Canadian hospitals.

It feels like ssi surveillance should work, but why? Is it all-inclusive data collection or the feedback to surgeons that counts? Would it be just as effective to guess at SSI rates and present them to surgeons anyway? Perhaps, but that would be unacceptable medicolegally. A more cost-effective method may be to present to surgeons absolute numbers (rather than incidences of SSI) compared with their absolute numbers from the year before. This could avoid the resource expenditure needed to track all operations, yet stimulate the surgeons' desire to improve their results. Although calculation of SSI rates has been accepted and promoted in academic circles, we have yet to prove that this tool actually improves the quality of patient care. More critical analysis and longer follow-up are needed. Taylor and colleagues should be commended for their honest reporting and encouraged to publish their results again in five years for a more longitudinal view of the effects of their ssi surveillance program.

\section{REFERENCES}

1. Olson M, O'Connor M, Schwartz ML. Surgical wound infections. Ann Surg 1984;199:253-65.

2. Sherertz RJ, Garibaldi RA, Marosok RD, et al. Consensus paper on the surveillance of surgical wound infections. Am J Infect Control 1992;20:263-70.

3. Law DJW, Mishriki SF, Jeffery PJ. The importance of surveillance after discharge from hospital in the diagnosis of postoperative wound infection. Ann R Coll Surg Engl 1990;72:207-9.

4. Olson MM, Lee JT. Continuous 10-year wound infection surveillance: results, advantages, and unanswered questions. Arch Surg 1990;125:794-803.

5. Lee JT. Wound infection surveillance. Infect Dis Clin North Am 1992;6:643-56.

6. Classen DC, Evans RS, Pestotnik SL, Horn SD. Menlove RL, Burke JP. The timing of prophylactic administration of antibiotics and the risk of surgical-wound infection. N Engl J Med 1992;326:281-6.

John MA Bohnen MD FRCSC FACS The Wellesley Hospital Toronto, Ontario 


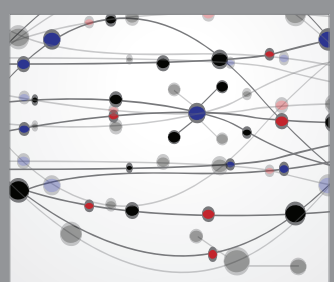

The Scientific World Journal
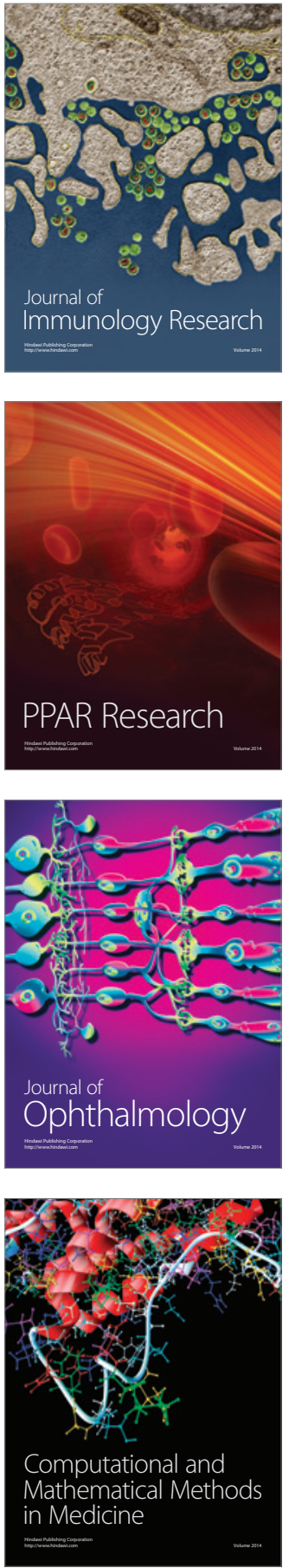

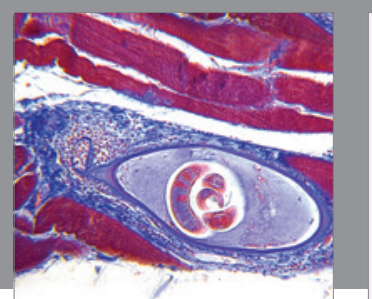

Gastroenterology Research and Practice

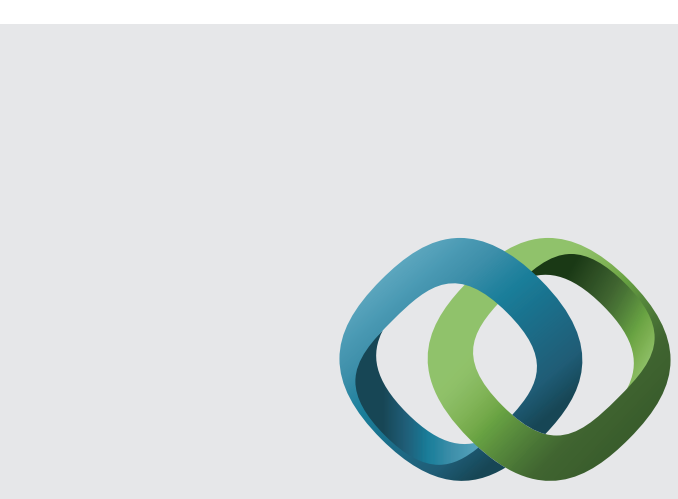

\section{Hindawi}

Submit your manuscripts at

http://www.hindawi.com
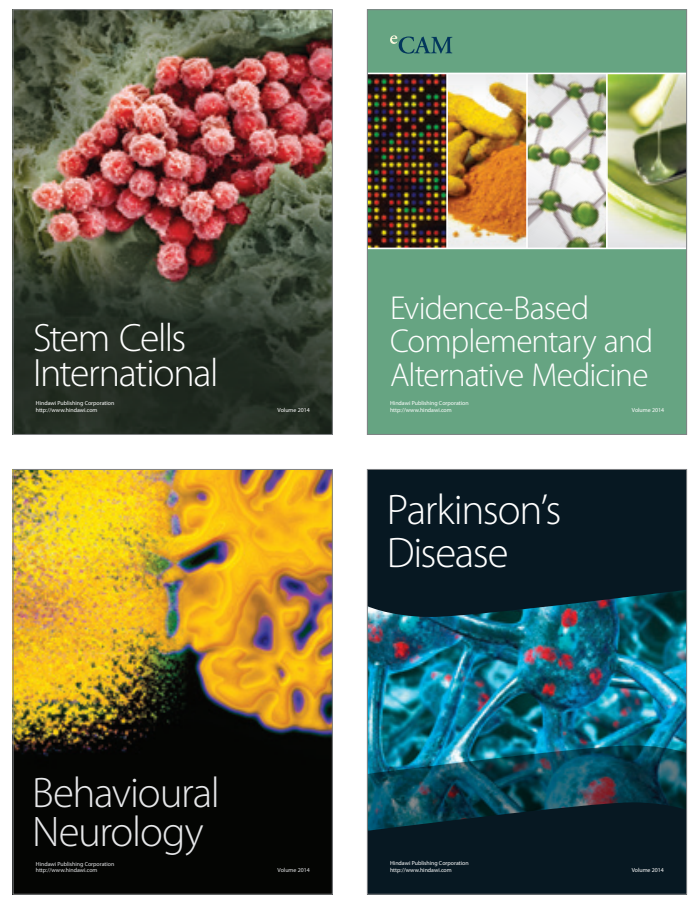
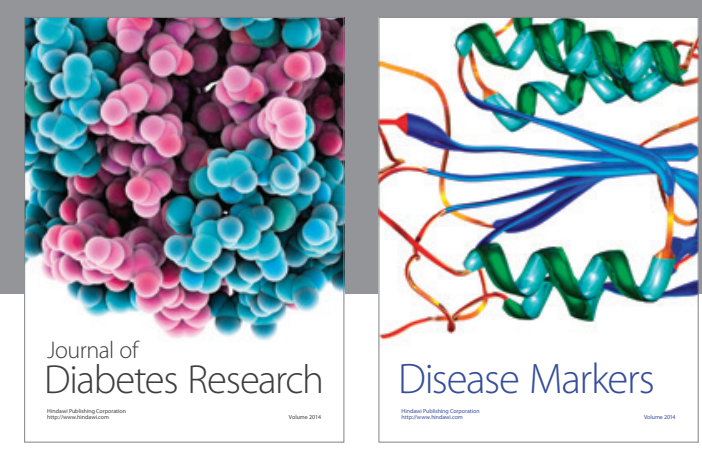

Disease Markers
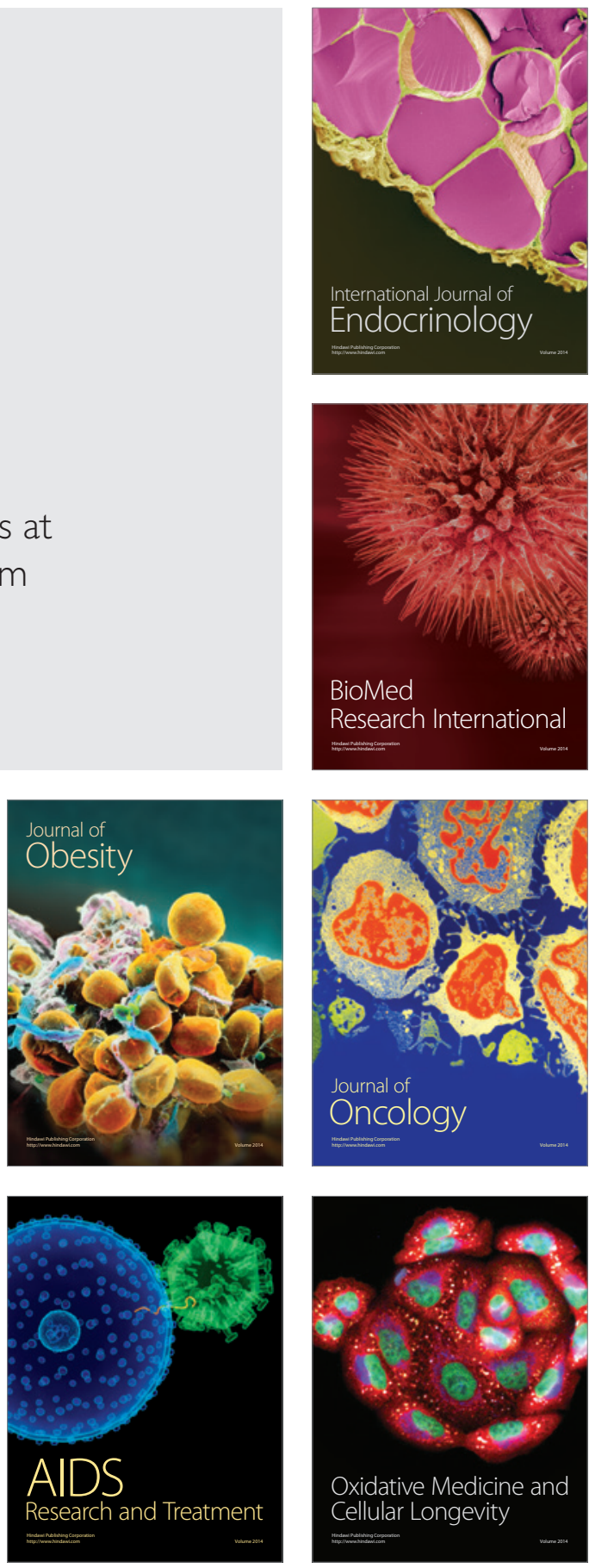